patterns over 3 consecutive years, patterns have been defined as: Persistent Long Quiescent (pLQ), Persistent Relapsing-Remitting (pRR), Persistent Chronic Active $(p C A)$ and Mixed, at least 2 different pattern types. Predictors of $p C A$ (vs. pLQ, $\mathrm{pRR}$ and mixed) and $\mathrm{pLQ}$ (vs. pCA, pRR and mixed) were identified by univariate and multivariate logistic regression analyses. Several baseline demographics (age, sex, ethnicity, disease duration, tobacco use, years of education and combined annual family income), disease characteristics at baseline (SLEDAI, PGA) and treatment categories (hydroxychloroquine, prednisolone and cytotoxic treatment followed at $\geq 75 \%$ of visits vs $<75 \%$ of visits) were used as independent variables. Results: 916 patients were identified. The results of the univariate analyses for pCA are shown in table 1. In the multivariate model, African American ethnicity (OR: $2.43,95 \%$ Cl: 1.19-4.94, p: 0.01) and high baseline SLEDAI (OR: 1.09, 95\% $\mathrm{Cl}: 1.03-1.16$, p: 0.004$)$ remained significant predictors of pCA. Higher education (>12 years; OR. 2.16, 95\% Cl: $1.11-4.20, \mathrm{p}: 0.02$ ) and low baseline SLEDAI (OR: $0.62,95 \% \mathrm{Cl}: 0.52-0.75, \mathrm{p}:<0.001$ ) were significant predictors of $\mathrm{pLQ}$ in the multivariate analysis while African American ethnicity (OR: $0.36,95 \% \mathrm{Cl}$ : $0.16-0.78, \mathrm{p}: 0.01$ ) and female patients (OR: $0.26,95 \% \mathrm{Cl}: 0.12-0.56, \mathrm{p}: 0.001$ ) were less likely to achieve persistent long quiescence.

Table 1. pCA (vs. pLQ, RR and mixed)

\begin{tabular}{lcc}
\hline & OR $(95 \%$ Cl) & p-value \\
\hline Sex (female vs. male) & $1.6(0.4-6.7)$ & 0.54 \\
Age ( $>40$ vs. $\leq 40$ yrs) & $1.4(0.7-2.8)$ & 0.32 \\
Disease Duration & $1.0(0.9-1.1)$ & 0.17 \\
African American (vs. other) & $2.6(1.3-5.3)$ & 0.007 \\
Years of education ( $>12$ vs. $\leq$ yrs) & $0.7(0.3-1.4)$ & 0.27 \\
Income & Ref & \\
$\quad<30000$ & $0.4(0.2-1.0)$ & 0.06 \\
$30000-65000 \$$ & $0.5(0.2-1.2)$ & 0.12 \\
$\geq 65000 \$$ & $2.1(1.0-4.6)$ & 0.06 \\
Smoking baseline (yes vs. no) & $1.1(1.0-1.2)$ & 0.002 \\
SLEDAI baseline & $1.5(1.0-2.2)$ & 0.04 \\
PGA baseline & $1.1(0.5-2.3)$ & 0.85 \\
Cytotoxic therapy $(\geq 75 \%$ vs. $<75 \%$ of visits) & $0.5(0.3-1.0)$ & 0.06 \\
Antimalaria therapy $(\geq 75 \%$ vs. $<75 \%$ of visits) & $1.1(0.6-2.2)$ & 0.82 \\
Prednisolone therapy $(\geq 75 \%$ vs. $<75 \%$ of visits) & & \\
\hline
\end{tabular}

Conclusions: In this large SLE cohort, African American ethnicity and high disease activity at the time of diagnosis are predictors of chronic activity, regardless of treatment, even after adjustment for education years and income, while higher education and low disease activity at baseline predict long-term quiescence.

References:

[1] Györi N, et al. Disease activity patterns over time in patients with systemic lupus erythematosus - Analysis of the Hopkins Lupus Cohort. Lupus Sci Med. 2017. In press.

Disclosure of Interest: None declared

DOI: 10.1136/annrheumdis-2017-eular.6861

\section{OP0046 RISK FACTORS FOR ADVERSE PREGNANCY OUTCOME IN ANTIPHOSPHOLIPID ANTIBODIES CARRIERS: RESULTS FROM A MULTICENTER ITALIAN COHORT OVER 20 YEARS OF EXPERIENCE}

M.G. Lazzaroni ${ }^{1}$, L. Andreoli ${ }^{1}$, C.B. Chighizola ${ }^{2}$, T. Del Ross ${ }^{3}$, M. Gerosa ${ }^{2}$, A. Kuzenko ${ }^{3}$, M.-G. Raimondo ${ }^{2}$, A. Lojacono ${ }^{1}$, F. Ramazzotto ${ }^{1}$, S. Zatti ${ }^{1}$, L. Trespidi ${ }^{2}$, P.L. Meroni ${ }^{2}$, V. Pengo ${ }^{3}$, A. Ruffatti ${ }^{3}$, A. Tincani ${ }^{1}$. ${ }^{1}-$, Brescia; ${ }^{2}$, Milano; ${ }^{3}$-, Padova, Italy

Background: Antiphospholipid antibodies (aPL) are risk factors for Adverse Pregnancy Outcome (APO). In particular, for aPL carriers (patients with aPL positivity with no thrombotic or obstetric history) pregnancy outcome and treatment are still undefined.

Objectives: To review pregnancy outcome in a large multicenter cohort of aPL carriers patients, according to different serological profile and treatment protocols. Methods: We reviewed 69 pregnancies in 61 patients, prospectively followed in 3 Italian centers (1994-2015). Patients with any complication in previous pregnancies or concomitant autoimmune diseases were excluded. APL profile was defined as the combination of the 3 criteria tests for aPL (Lupus Anticoagulant, anti-cardiolipin, anti-Beta2 Glycoprotein I). APO was defined as: miscarriage $(<10 w)$, fetal death $(\geq 10 w)$, severe preterm delivery $(\leq 34 w)$ with or without preeclampsia (PE), HELLP syndrome or perinatal death.

Results: Regarding aPL profile: $44(64 \%)$ were single positive, $16(23 \%)$ were double and $9(13 \%)$ were triple. aPL non-criteria manifestations were present in $6(9 \%)$ and lupus-like manifestations in $5(7 \%)$. Eight pregnancies had a combination treatment with prophylactic low molecular weight heparin (LMWH) plus low dose aspirin (LDA), 37 had LDA alone and 24 had no treatment. We observed 2 thrombotic events (3\%, 1 deep venous thrombosis and 1 ischemic stroke) and 6 APO (9\%): 3 fetal deaths and 3 pre-term birth $\leq 34$ w with PE. We compared 8 complicated (6 APO,2 thrombosis) with 61 non-complicated pregnancies (Table 1). Acquired risk factors ( $p: 0.006)$, non-criteria aPL $(p: 0.018)$ and lupus-like manifestation ( $\mathrm{p}: 0.010)$, triple positive aPL profile ( $\mathrm{p}: 0.001)$ were associated with APO. The combination treatment was also more frequent in APO pregnancies ( $\mathrm{p}: 0.005)$.

\begin{tabular}{|c|c|c|c|c|}
\hline & $\begin{array}{c}\text { Complicated } \\
\text { pregnancies }(n=8)\end{array}$ & $\begin{array}{l}\text { Non-complicated } \\
\text { pregnancies }(n=61)\end{array}$ & P-value ^ & OR $(95 \% \mathrm{Cl})$ \\
\hline $\begin{array}{l}\text { Mean age at pregnancy } \\
\text { (mean (SD)) }\end{array}$ & $28.3(5.8)$ & $32.7(4.6)$ & $0.015^{5}$ & \\
\hline Acquired risk factors ${ }^{2}$ & $6(75 \%)$ & $14(23 \%)$ & 0.006 & $10.7(1.55 \cdot 82.4)$ \\
\hline Congenital thrombophilia & $2(25 \%)$ & $4(7 \%)$ & 0.140 & \\
\hline $\begin{array}{l}\text { Non criteria aPL } \\
\text { manifestations : }\end{array}$ & $3(38 \%)$ & $3(5 \%)$ & 0.018 & $11.6(1.37-108)$ \\
\hline Lupus-like manifestations & $3(38 \%)$ & $2(3 \%)$ & 0.010 & $17.7(1.77-213)$ \\
\hline Single positive & $3(38 \%)$ & $41(67 \%)$ & 0.128 & \\
\hline Double positive & $0(0 \%)$ & $16(26 \%)$ & 0.183 & \\
\hline Triple positive & $5(63 \%)$ & $4(7 \%)$ & 0.001 & $23.7(3.22-211)$ \\
\hline No treatment & $1(13 \%)$ & $23(38 \%)$ & 0.246 & \\
\hline LDA & $3(38 \%)$ & $34(56 \%)$ & 0.457 & \\
\hline LDA+LMWH & $4(50 \%)$ & $4(7 \%)$ & 0.005 & $14.2(1.98-116)$ \\
\hline
\end{tabular}

Table 1. Complicated pregnancies (APO and thrombosis) compared with non-complicated pregnancies univariate analysis Test 5 Student's T- test

Acquired nskfactors Obesin (BM Nonstenemia, diabetes mellitus

Non-citeria aPL manifestations were defined as the presence of at least one of the followings:

Conclusions: Acquired risk factors, aPL profile (triple positivity), lupus-like and non-criteria aPL manifestations could represent risk factors for pregnancy complications and could determine failure to conventional treatment.These patients may deserve additional treatment, for example LMWH at higher/anticoagulant dose or immunomodulatory treatment (e.g.hydroxychloroquine, also considering its anti-platelet/anti-thrombotic effects).

Disclosure of Interest: None declared

DOI: 10.1136/annrheumdis-2017-eular.2260

\section{WEDNESDAY, 14 JUNE 2017 Osteoporosis treatment gap, new options and new strategies}

\section{OP0047 OSTEOPOROTIC HIP FRACTURE PREVENTION: ARE WE IN A CRISIS?}

M. Sehgal ${ }^{1}$, A. Mithal ${ }^{2}$, A. Mithal ${ }^{3}$, G. Singh ${ }^{4} .{ }^{1}$ Student, Menlo Atherton High School, Atherton, United States; ${ }^{2}$ Student, IIIT Delhi, Delhi, India; ${ }^{3}$ Epidemiology, ICORE, Woodside; ${ }^{4}$ Gastroenterology and Hepatology, Stanford University, Stanford, United States

Background: Osteoporotic hip fractures are a significant cause of morbidity and mortality in the elderly. With the aging of the US population, it was expected that there would be a nationwide "epidemic" of osteoporotic fractures; however, Medicare reimbursement for osteoporosis screening and the rapid uptake of bisphosphonate therapy in the late 1990s and early 2000s probably forestalled this epidemic. In fact, osteoporotic hip fractures numbers declined during this time, despite the dramatic growth of the elderly population (1). However, bisphosphonate prescriptions have dropped precipitously by over $50 \%$ from their peak in 2008 (2), likely because of concerns regarding their adverse events. In this era of "crisis" in osteoporosis prevention, has the pendulum swung back on hip fracture numbers?

Objectives: To study the number and prevalence of osteoporotic hip fractures in patients 50 years and older over the last 22 years (1993-2014) in the US?

Methods: The Nationwide Inpatient Sample (NIS) is a stratified random sample of all US community hospitals. It is the only US national hospital database with information on all patients, regardless of payer, including persons covered by Medicare, Medicaid, private insurance, and the uninsured. We examined all inpatient hospitalizations in NIS from 1993 to 2014 with a primary diagnosis of non-traumatic hip fractures, and calculated age-adjusted prevalence rates. We excluded patients with concomitant diagnosis of major trauma, primary or secondary tumors locally, osteopetrosis and procedures for revision surgery or surgery for complications.

US population estimates and projections for the resident US population were obtained from the US Census Bureau.

Results: There were 407 million all-cause hospitalizations in 1.88 billion personyears of observation among people 50 years of older from 1993 to $2014(21,626$ hospitalizations per 100,000 person-years). During this time-period, there were 6.15 million hospitalizations for osteoporotic hip fractures in this population (327 per 100,000 person-years). After an initial increase in hip fractures from 1993 to 1996 , there was a decline of $10.6 \%$ in total number of hip fractures from 300,154 in 1996 to 268,356 in 2010 even though population of 50 years and older increased from 69.9 million to 99.6 million in the same time. After bottoming out in 2010 , the total number of hip fractures increased by $6.2 \%$ to 285,050 in 2014 (Figure, blue broken line). However, the age-standardized prevalence rate of hip fractures declined steadily (from 414/100,000 in 1996 to 269/100,000 in 2014, $p<0.0001$, Figure solid red line), although the rate of decline has ameliorated somewhat from 2010 onwards (not statistically significant).

Conclusions: The total number of osteoporotic hip fractures in 50 years and 
Total number and prevalence of osteoporotic hip fractures in the US (1993-2014)

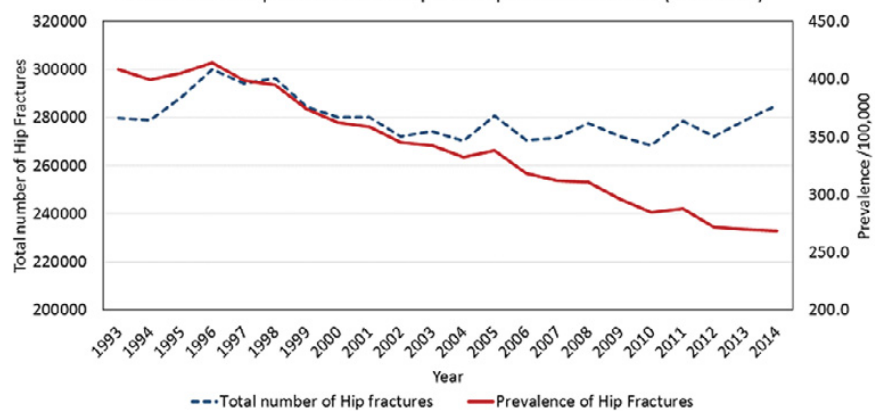

older declined since 1996, bottomed out in 2010 but has started increasing again. It is critical to evaluate risks and benefits of preventive treatments for optimal management of this potentially serious problem.

\section{References:}

[1] Sehgal, A., et al., A victory in the war on osteoporosis? Declining prevalence of hospitalizations for non-traumatic hip fractures in the US. Ann Rheum Dis 2008;67(Suppl II):55.

[2] Wysowski, D.K. and P. Greene, Trends in osteoporosis treatment with oral and intravenous bisphosphonates in the United States, 2002-2012. Bone, 2013. 57(2): p. 423-8.

Disclosure of Interest: None declared

DOI: 10.1136/annrheumdis-2017-eular.5685

\section{OP0048 ROMOSOZUMAB RAPIDLY REDUCES CLINICAL VERTEBRAL FRACTURE INCIDENCE: RESULTS FROM THE FRAME STUDY}

P. Geusens ${ }^{1,2}$, M. Oates ${ }^{3}$, A. Miyauchi ${ }^{4}$, J. Adachi ${ }^{5}$, M. Lazaretti-Castro ${ }^{6}$, P.R. Ebeling ${ }^{7}$, C.A. Perez Nino ${ }^{8}$, C.J. Milmont ${ }^{9}$, A. Grauer ${ }^{9}$, C. Libanati ${ }^{10}$ ${ }^{1}$ Maastricht UMC, Maastricht, Netherlands: ${ }^{2}$ UHasselt and ReumaClinic, Genk, Belgium; ${ }^{3}$ Pacific Central Coast Health Center, Santa Maria, United States; ${ }^{4}$ Miyauchi Medical Center, Osaka, Japan; ${ }^{5}$ McMaster University, Hamilton, Canada; ${ }^{6}$ IMA Brazil, São Paulo, Brazil; ${ }^{7}$ Monash University, Clayton, Australia; ${ }^{8}$ UNIENDO, Bogota, Colombia; ${ }^{9}$ Amgen, Thousand Oaks, United States; ${ }^{10}$ UCB, Brussels, Belgium

Background: Romosozumab (ROMO) inhibits sclerostin and has a dual effect on bone, increasing formation while decreasing resorption, resulting in significant increases in bone mineral density (BMD) at 6 months $(\mathrm{m})$, which at $12 \mathrm{~m}$ reach $13.3 \%$ vs placebo (PBO) at the spine. ${ }^{1}$ Using high resolution quantitative computed tomography, BMD increases were observed at both trabecular and cortical compartments of the spine, explaining the significant reductions in radiographic vertebral fracture (VFx) risk in women with osteoporosis (OP) enrolled in the FRAME trial (NCT01575834).

Objectives: Here, we report the effect of ROMO on clinical (clin) VFx incidence over $12 \mathrm{~m}$ in women in FRAME with back pain.

Methods: FRAME enrolled 7180 postmenopausal women with OP, mean age 70.9 yrs (total hip T-score -2.5 to -3.5 ) and no severe VFx. Patients received monthly ROMO ( $\mathrm{n}=3589 ; 210 \mathrm{mg})$ or PBO $(\mathrm{n}=3591)$ for $12 \mathrm{~m}$. At monthly visits, women with back pain consistent with a clin VFx had a confirmatory spinal X-ray. Clin VFx risk (ROMO vs PBO) was calculated by Cox-proportional hazards model. Results: Of 119 women reporting back pain over $12 \mathrm{~m}, 20$ women were diagnosed with a new or worsening VFx. With ROMO, 3 clin VFx $(<0.1 \%$; all at $<2 \mathrm{~m})$ were identified vs $17(0.5 \%$ at $12 \mathrm{~m})$ with PBO (Figure). Clin VFx risk was $83 \%$ lower in the ROMO group vs $\mathrm{PBO}$ at $12 \mathrm{~m}$ (hazard ratio $0.17 ; 95 \% \mathrm{Cl}, 0.05-0.58 ; \mathrm{p}=0.001$ ). In women with clin VFx vs no clin VFx, the lumbar spine T-score was numerically lower and the FRAX score higher at baseline; other baseline characteristics were comparable among all women who reported back pain.

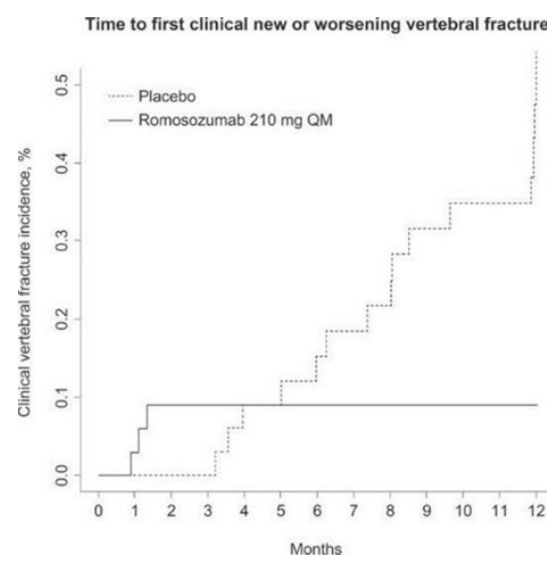

Conclusions: $\mathrm{ROMO}$ treatment for $12 \mathrm{~m}$ was associated with rapid and large reductions in clin VFx risk vs PBO. In the ROMO group, all clin VFx occurred $<2 \mathrm{~m}$; clin VFx risk was $\geq 5$ times higher with $\mathrm{PBO}$ vs ROMO. Monthly study visits in FRAME allowed for timely radiologic confirmation of a suspected clin VFX.

References:

[1] Cosman F et al. N Engl J Med 2016;375:1532-43.

Acknowledgements: Funded by Amgen Inc. and UCB Pharma.

Disclosure of Interest: P. Geusens Grant/research support from: Amgen, Pfizer, MSD, UCS, Abbott, Eli Lilly, BMS, Novartis, Roche, Will-Pharma, Consultant for: Amgen, Speakers bureau: Amgen, M. Oates Grant/research support from: Amgen, Speakers bureau: Amgen, Eli Lilly, A. Miyauchi Consultant for: Amgen, Astellas BioPharma, J. Adachi Grant/research support from: AbbVie, Amgen, Eli Lilly, Merck, Pfizer, Consultant for: Amgen, Merck, Speakers bureau: Amgen, M. Lazaretti-Castro Grant/research support from: Amgen, Consultant for: Amgen, Eli Lilly, Sanofi, Speakers bureau: Sanofi, P. Ebeling Grant/research support from: Amgen, Merck, Eli Lilly, Consultant for: Amgen, Eli Lilly, Radius, C. A. Perez Nino Shareholder of: UNIENDO - Clinical Research, Employee of: UNIENDO Clinical Research, C. Milmont Shareholder of: Amgen, Employee of: Amgen, A. Grauer Shareholder of: Amgen, Employee of: Amgen, C. Libanati Shareholder of: UCB Pharma, Employee of: UCB Pharma

DOI: 10.1136/annrheumdis-2017-eular.5796

\section{OP0049 SYSTEMATIC REVIEW OF RANDOMIZED CONTROLLED TRIALS EVALUATING BISPHOSPHONATES FOR THE PREVENTION AND TREATMENT OF GLUCOCORTICOID- INDUCED OSTEOPOROSIS}

A. Makhzoum ${ }^{1}$, L. Petriw ${ }^{2}$, M. Sattin ${ }^{3}$, T. Towheed ${ }^{4} .{ }^{1}$ Department of Rheuamtology; ${ }^{2}$ Department of Internal medicine, Queen's University, Kingston; ${ }^{3}$ Department of Internal medicine, University of Western Ontario, London;

${ }^{4}$ Department of Rheumatology, Queen's University, Kingston, Canada

Background: Glucocorticoid therapy is a major risk factor for osteoporosis related fractures. A previous meta-analysis conducted by Homik et al reported that bisphosphonates therapy increased BMD in glucocorticoid- induced osteoporosis (GIO) when compared to placebo, whereas results for incident vertebral fracture did not reach statistical significance

Objectives: To evaluate the efficacy of bisphosphonates in GIO based on randomized controlled trials (RCTs). Both placebo controlled and active comparator trials were analyzed.

Methods: Two authors screened citations from the following electronic databases: Medline (1998-2015), EMBASE (1998-2015), Cochrane Library (1998-2015). A manual search was completed for conference proceedings from the ACR (2010-2015), CRA (2009-2015), and ASBMR (2009-2014). We used the study by Homik et al to identify RCTs published prior to 1998 . Only RCTs that had a minimum prednisone dosage of $5 \mathrm{mg} /$ day or equivalent and treatment duration of at least 3 months were included. Primary outcomes were changes in BMD and incident fractures. Two authors abstracted data using a standardized data abstraction form. We used the Cochrane Risk of Bias Tool to evaluate the quality of the selected RCTs and devised a quality score ranging from 0 to 6 , where 6 represents the highest quality.

Results: A total of 466 citations were identified (239 Medline, 217 EMBASE, and 10 Cochrane Library). Fourteen RCTs met the inclusion criteria. An additional two RCTs were identified from conference proceedings. Eleven RCTs compared bisphosphonates to a placebo, three RCTs compared bisphosphonates to a vitamin $\mathrm{D}$ derivative, one RCT compared alendronate to teriparatide, and one RCT compared zoledronic acid to risedronate. The RCTs were of reasonably good quality with a mean quality score of 4 .

Overall, of the 11 RCTs that compared bisphosphonates to a placebo, all found that the bisphosphonates were superior. Nine RCTs were pooled for mean percentage change in lumbar spine BMD (bisphosphonates $n=667$, placebo $\mathrm{n}=654$ ). The pooled mean percentage change was in favor of bisphosphonates compared to placebo [weighted mean difference (WMD) of $4.03 \%, 95 \% \mathrm{Cl}$ (1.59-6.47), $p=0.001]$. Six RCTs were pooled for mean percentage change in femoral neck BMD (bisphosphonates $n=486$, placebo $n=481$ ) and the results favored bisphosphonates compared to placebo [WMD of $2.95 \%, 95 \% \mathrm{Cl}$ (0.09 -5.82), $P=0.04]$. Seven RCTs were pooled for outcome of incident fractures (bisphosphonates $n=613$, placebo $n=469$ ) and the results favored bisphosphonates compared to placebo [RR of $0.65,95 \% \mathrm{Cl}(0.48-0.88)$, $\mathrm{P}=0.006$ ] (Figure 1). Results were pooled using RevMan (version 5.3).

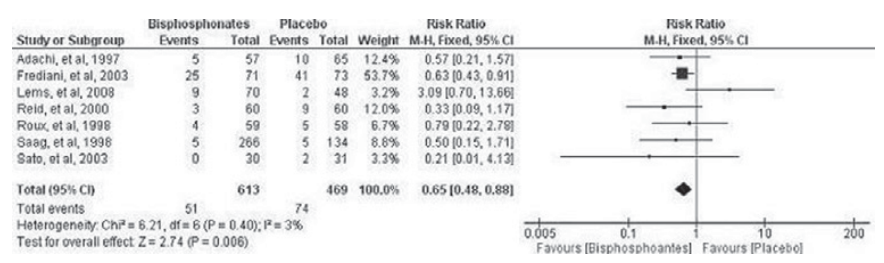

Figure 1

Conclusions: Bisphosphonates mitigate adverse changes in BMD and lower fracture risk in patients treated with glucocorticoids. 